\title{
Mezcal as a Novel Source of Mixed Yeasts Inocula for Wine Fermentation
}

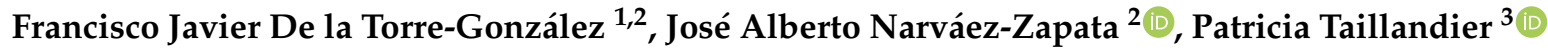 \\ and Claudia Patricia Larralde-Corona $2, * \mathbb{D}$ \\ 1 Innovak Global, Investigación y Desarrollo, Lab. de Biología Molecular (Innovak Global, R\&D, Molecular \\ Biology Lab), Blvd Vicente Lombardo Toledano \#6615 Col. La Concordia, C.P. 31375 Chihuahua, \\ Chihuahua, Mexico; fdelatorre@innovakglobal.com \\ 2 Instituto Politécnico Nacional, Centro de Biotecnología Genómica, Laboratorio de Biotecnología \\ Industrial (National Polytechnic Institute, Center for Genomic Biotechnology, Industrial Biotechnology Lab), \\ Blvd del Maestro s/n esq. Elías Piña Col. Narciso Mendoza, C.P. 88710 Reynosa (Tamaulipas), Mexico; \\ jnarvaez@ipn.mx \\ 3 Laboratoire de Génie Chimique, Université de Toulouse (Chemical Engineering Lab, Toulouse University), \\ CNRS, INPT, UPS, 31432 Toulouse CEDEX 4, France; patricia.taillandier@ensiacet.fr \\ * Correspondence: plarralde@ipn.mx; Tel.: +52-(55)-57296000 (ext. 87726)
}

Received: 9 September 2020; Accepted: 12 October 2020; Published: 16 October 2020

check for updates

\begin{abstract}
Mezcal yeasts were evaluated for their potential as grape-juice fermenters, characterizing their fermentation performance, both in terms of primary and volatile metabolites. Experiments were first carried-out in a semi-synthetic medium and then on grape juice, and population dynamics of the chosen mixed inoculum was assessed in grape juice. Accordingly, we initially tested 24 mezcal yeasts belonging to ten different species, and chose those that were more productive and stress tolerant for the mixed (dual) inoculum, having a final selection of three Saccharomyces cerevisiae strains (plus Fermichamp, a commercial wine strain) and three non-Saccharomyces strains, belonging to Kluyveromyces marxianus, Torulaspora delbrueckii, and Zygosaccharomyces bailii species. For the combination S. cerevisiae/T. delbrueckii (Sc/Td) mixed inoculum, we observed increasing isoamyl alcohol and phenyl ethyl acetate concentrations, as compared with the use of individual Saccharomyces strains, which resulted in a fruitier aroma profile. Alcohol final concentration was in average lower for the Sc/Td inoculum (fermentation power, FP, 13.6) as compared with the individual mezcal Saccharomyces strains (FP 14.3), and it was the highest when Td was co-cultured with the commercial strain (FP 14.6). Overall, our results show the feasibility of using yeasts isolated from mezcal as a novel source of inoculum for wine-type fermentation.
\end{abstract}

Keywords: yeasts mixed inoculum; mezcal; Saccharomyces; non-Saccharomyces

\section{Introduction}

Worldwide, two main phenomena are increasingly being reported for wine fermentation, first is the occurrence of stuck fermentations, as fructose becomes the main carbohydrate during the late stages of alcoholic fermentation, and the yeasts have to ferment under conditions of high ethanol concentration and nitrogen limitation. And second, a higher alcohol content and less aroma complexity [1,2]. Some authors have used the positive response to a certain stress as a selection tool for yeast with potential for use in wine production. For example, Zuzuarregui and del Olmo [3] analyzed the resistance to oxidative, osmotic and ethanol stresses among a collection of commercial (winery) and non-commercial S. cerevisiae strains, and correlating fermentative behavior with resistance to oxidative stress (by exposure to $\mathrm{H}_{2} \mathrm{O}_{2}$ ) and to ethanol stress as the most relevant. García and collaborators [4] analyzed the tolerance to osmotic pressure, ethanol, and $\mathrm{pH}$ stresses in a warm climate region $\mathrm{DO}$ 
'Vinos de Madrid' (Spain). These authors identified some Saccharomyces and non-Saccharomyces strains adapted to these fermentation stresses, concluding that these yeasts are important in the quality wine in these warm areas.

The use of non-conventional yeast as inoculum for wine making has increased in importance in the last decades, as it has been observed that some strains can increase the aroma complexity of the fermented products [5]. Our research has explored the potential use of novel yeast strains obtained from mezcal, which is a traditional Mexican liquor that involves a very stressful alcoholic fermentation [6]. These yeasts are proposed since the musts of cooked agave plants contain a high fructose content (around 90\% of fermentable sugars), Maillard compounds, furfural and even toxic saponins, and mezcal fermentation is carried out without temperature control, making this a very stressful fermentation system [2]. In addition, these yeasts are part of a different domestication event as compared to other S. cerevisiae wine strains [7], and their phenotypic characteristics may be different, particularly, when submitted to high fructose concentration [6,8,9]. Novel yeast applications to increase the aroma complexity in wine may be supported by the used of mixed starters. As an example, co-inoculation of $S$ cerevisiae strains changes wine composition regarding to monoculture [1] and mixed starters with Saccharomyces and non-Saccharomyces (Hanseniaspora vineae) strains enhances aromatic profile compared to simple mixed inoculation and increased the wine quality [10].

In the current study, fermentative profiles of mezcal yeasts (Saccharomyces and non-Saccharomyces) in terms of primary and volatile metabolites production were compared when cultivated in a semi-synthetic medium (M3) to simulate wine fermentation. The results were used to choose Saccharomyces and non-Saccharomyces strains to be cultivated in grape juice individually, or as a mixed inoculum, and their fermentative performance and potential as starters for wine production were evaluated.

\section{Materials and Methods}

\subsection{Yeast Strains and Inoculum Growth Conditions}

The 24 yeast strains used belong to the mezcal LCBG yeast collection (which comprises 96 different strains, belonging to ten different yeast genera) and are conserved in $60 \%$ glycerol at $-70{ }^{\circ} \mathrm{C}$. The commercial wine strain Saccharomyces cerevisiae Fermichamp (DSM Food Specialties B.V., The Netherlands) was used as a control for its fructophilic character, which is used to reactivate stuck fermentations as indicated by the manufacturer. The strains used were selected based on both their level of stress tolerance [6] and to be representative of the yeast diversity found in the fermentation of mezcal from Tamaulipas (Mexico). For all the strains, their 26S nucleotide sequences are available in the GenBank and are presented on Table 1, along with the fermentation stage from where they were originally isolated.

Table 1. Molecular identification and mezcal fermentation stage of isolation of the yeasts used in this study.

\begin{tabular}{cccc}
\hline Species & Strain ID & $\begin{array}{c}\text { GenBank Accession } \\
\text { Number }\end{array}$ & $\begin{array}{c}\text { Fermentation Stage of } \\
\text { Isolation * }\end{array}$ \\
\hline & $\begin{array}{c}\text { Fermichamp } \\
\text { Sc3Y2 }\end{array}$ & JQ824877 & - \\
& Sc3Y3 & JQ824872 & Final \\
& Sc3Y4 & JQ824875 & Final \\
& Sc3Y5 & JQ824869 & Final \\
& Sc3Y8 & JQ824874 & Final \\
Saccharomyces cerevisiae & Scmosca3 & KT945088 & Fruit fly on the vat surface \\
& Sc3D6 & JQ824876 & Final \\
& Sc3D5 & KT945085 & Final \\
& Sc3D4 & KT945086 & Final \\
& Sc3D2 & JQ824871 & Final \\
& Sc4Y3 & KT945087 & Trapiche \\
\hline
\end{tabular}


Table 1. Cont.

\begin{tabular}{|c|c|c|c|}
\hline Species & Strain ID & $\begin{array}{c}\text { GenBank Accession } \\
\text { Number }\end{array}$ & $\begin{array}{l}\text { Fermentation Stage of } \\
\text { Isolation * }\end{array}$ \\
\hline \multirow{3}{*}{ Kluyveromyces marxianus } & Km4D3 & KT945094 & Trapiche \\
\hline & Km1D5 & KT945093 & Trapiche \\
\hline & Km1Y9 & KT945092 & Early \\
\hline \multirow{3}{*}{ Torulaspora delbrueckii } & Td1AN9 & KT945090 & Early \\
\hline & Td1AN2 & KT945089 & Early \\
\hline & Td1AN1 & KT945091 & Early \\
\hline Pichia kluyveri & Pk4D6 & KT945083 & Trapiche \\
\hline Yamadazyma mexicana & Pm1AN3 & KT945081 & Early \\
\hline Meyerozyma guilliermondii & Pg1Y12 & KT945082 & Early \\
\hline Clavispora lusitaniae & $\mathrm{Cl} 4 \mathrm{Y} 4$ & KT945080 & Trapiche \\
\hline Candida parapsilosis & Cp1Y7 & KT945079 & Early \\
\hline Rhodotorula mucilaginosa & $\mathrm{RmP12}$ & KT945095 & Early \\
\hline Zygosaccharomyces bailii & Zb3Y1 & KT945084 & Final \\
\hline
\end{tabular}

An initial preculture of the tested yeasts was grown on yeast extract-peptone-dextrose agar YPD, Difco Laboratories, France) agar plates containing $10 \mathrm{~g} / \mathrm{L}$ yeast extract, $20 \mathrm{~g} / \mathrm{L}$ peptone, $20 \mathrm{~g} / \mathrm{L}$ D-glucose, plus $20 \mathrm{~g} / \mathrm{L}$ bacteriological agar (Difco Laboratories, France), and incubated at $30^{\circ} \mathrm{C}$ for $48 \mathrm{~h}$. A loop of this preculture was used as inoculum for liquid YPD broth incubated for $24 \mathrm{~h}$ at $30^{\circ} \mathrm{C}$ with shaking at $200 \mathrm{rpm}$, and final yeast concentrations (total and viable) were quantified using a Neubauer chamber, adjusting if needed using sterile isotonic solution $(9 \mathrm{~g} / \mathrm{L} \mathrm{NaCl}$ solution) and used immediately as inoculum at in the fermentation experiments carried out as described below.

\subsection{Setup of Minifermentation Conditions}

All fermentation experiments were carried out in minibioreactor tubes of $50 \mathrm{~mL}$ with 4-hole vent caps (Corning Science de México, Reynosa, TAM, Mexico), but covering 3 of the 4 holes available in the cap with cellotape just before inoculation, to allow semianaerobic fermentation conditions and also to diminish loss of water. The minibioreactors contained $20 \mathrm{~mL}$ of either the semi-synthetic medium M3 (Oliva-Hernández et al., 2013) or grape juice medium. Medium M3 contained $200 \mathrm{~g} / \mathrm{L}$ of total sugars (glucose/fructose, 1:1), $1 \mathrm{~g} / \mathrm{L}$ of yeast extract, $2 \mathrm{~g} / \mathrm{L}$ of $\left(\mathrm{NH}_{4}\right)_{2} \mathrm{SO}_{4}, 0.4 \mathrm{~g} / \mathrm{L}$ of $\mathrm{MgSO}_{4} 7 \mathrm{H}_{2} \mathrm{O}, 5 \mathrm{~g} / \mathrm{L}$ of $\mathrm{KH}_{2} \mathrm{PO}_{4}$, dissolved distilled water and with the $\mathrm{pH}$ adjusted to 5 before autoclaving. For wine-type fermentations, pasteurized red grape juice was used (Carrefour, Toulouse, France), which was typically around $200 \mathrm{~g} / \mathrm{L}$ of total sugars, and also adding a small volume of ammonium sulfate sterile solution to have a final concentration of $2 \mathrm{~g} / \mathrm{L}$ of $\left(\mathrm{NH}_{4}\right)_{2} \mathrm{SO}_{4}$ in the grape juice, to avoid nitrogen limitation during fermentation. The inoculum used was $3 \times 10^{6} \mathrm{cells} / \mathrm{mL}$, either when using individual or a mixed inoculum. In the latter case, a ratio of $1: 9$ of $S$. cerevisiae/non-Saccharomyces strains was used. Incubation was performed at $30^{\circ} \mathrm{C}$ using an agitated Minitron HG incubator (Infors AG, Switzerland) at $75 \mathrm{rpm}$. Each experiment was run in triplicate, hence withdrawing and analyzing three different tubes per sampling time, and measurements for each minibioreactor were performed at least two times. Average values and standard deviation are reported accordingly.

\subsection{Mixed Yeasts Populations Quantification}

Quantification of the yeasts populations during fermentation was performed on Wallerstein Differential Agar WLD (Sigma-Aldrich, St. Louis, MO, USA) for following the non-Saccharomyces populations, as $S$. cerevisiae is unable to grow on such media. This allowed an easy verification of the viable count of the non-Saccharomyces species. Colony counts for S. cerevisiae were obtained by 
subtracting the WLD count number to the count obtained on on Wallerstein Nutrient Agar (WL Sigma-Aldrich, St. Louis, MO, USA) for whole yeasts population counts, and are reported as colony forming units per milliliter $\mathrm{CFU} / \mathrm{mL}$. Total and viable cell counts were determined by counting on a Neubauer chamber, using methylene blue staining as an indicator of viability of the whole population (Saccharomyces and non-Saccharomyces). All the samples were analyzed in triplicate.

\subsection{Biomass and $\mathrm{CO}_{2}$ Production Quantification}

Biomass was quantified as dry weight by centrifuging $2 \mathrm{~mL}$ of each sample in dry and pre-weighed $2 \mathrm{~mL}$ Eppendorf tubes for $10 \mathrm{~min}$ at 14,000 rpm. The supernatant was recovered and filtered for further high performance liquid chromatography HPLC analysis, and tubes containing the biomass pellet were dried half-open at $60^{\circ} \mathrm{C}$ overnight, placed in a desiccator for at least $4 \mathrm{~h}$, and then weighed. Biomass production was calculated as the difference in the weight of the tube divided by the volume of the centrifuged sample. Duplicate samples were taken from each of the three minibioreactor tubes per sampling time.

The release of carbon dioxide was used as an indicator of fermentation progress and to decide when to stop the experiments; hence, weight loss was followed for each minibioreactor every $24 \mathrm{~h}$. At the experimental conditions tested, both in the semi-synthetic medium M3 and in grape juice, the rate of water loss in the minibioreactors per open hole in the cap was measured to be $0.0034 \mathrm{~g}_{\text {water }} / \mathrm{h}$ per hole $\left(\mathrm{R}^{2}=0.999\right)$, and this value was used as a correction factor to assess the $\mathrm{CO}_{2}$ liberated per liter of medium.

\subsection{Sugar Consumption and Metabolite Quantification by HPLC}

The consumption of sugars and the production of metabolites (ethanol, glycerol and acetic acid) in the centrifuged ( $15 \mathrm{~min}$ at 10,000 $\mathrm{rpm}$ at $4{ }^{\circ} \mathrm{C}$ ) and filtered (Millex-GV13 $0.22 \mu \mathrm{m}$ pore size, Millipore Sigma, Burlington, MA, USA) sample supernatants were measured with an Accela HPLC (Thermo Scientific, France) coupled to an auto sampler and using a Phenomenex ROA-Organic acid column ( $250 \mathrm{~mm} \times 4.6 \mathrm{~mm} ; 8-\mu \mathrm{m}$ diameter beads). The mobile phase was $5 \mathrm{mM} \mathrm{H}_{2} \mathrm{SO}_{4}$. The volume of the injection loop was $25 \mu \mathrm{L}$ with each run lasting around $30 \mathrm{~min}$ with a flow rate of $0.17 \mathrm{~mL} / \mathrm{min}$ at $30^{\circ} \mathrm{C}$. The peaks were detected by infra red IRD and/or ultra violet UVD, depending on the compound measured. Calibration curves were constructed using ethanol, glycerol, acetic acid, fructose, and glucose standards ranging from 0.125 to $5 \mathrm{~g} / \mathrm{L}$.

\subsection{Volatile Compound Quantification by GC-MS}

The production of volatile metabolites relevant for the organoleptic profile characterization of each strain was assessed by GC-MS in a TraceGC machine (Thermo Finnigan, Villebon Sur Yvette, France). Fermentation samples were centrifuged at $7000 \mathrm{rpm}(5697 \times g)$ for $15 \mathrm{~min}$ at $10^{\circ} \mathrm{C}$ in a Sigma $6 \mathrm{~K} 15$ centrifuge, and $10 \mathrm{~mL}$ were taken and extracted by SPME (PDMS fiber assembly, SUPELCO, Bellefonte, $\mathrm{PE}, \mathrm{USA}$ ) at $40^{\circ} \mathrm{C}$ and adding $3 \mathrm{~g}$ of $\mathrm{NaCl}$, and the volatile compounds were measured by using a ZB-5ms Phenomenex column ( $30 \mathrm{~m}$ length $\times 0.25 \mathrm{~mm}$ internal diameter, $0.25 \mu \mathrm{m}$ film thickness). The carrier gas was helium at a flow rate of $1 \mathrm{~mL} / \mathrm{min}$, the injector was set at $240{ }^{\circ} \mathrm{C}$, and the following temperature program was used: $10 \mathrm{~min}$ at $35^{\circ} \mathrm{C}$, first ramp of $2{ }^{\circ} \mathrm{C} / \mathrm{min}$ up to $60^{\circ} \mathrm{C}$, isothermal at $60^{\circ} \mathrm{C}$ for one minute, second ramp of $2.5^{\circ} \mathrm{C} / \mathrm{min}$ up to $90^{\circ} \mathrm{C}$, third ramp of $10^{\circ} \mathrm{C} / \mathrm{min}$ up to $130^{\circ} \mathrm{C}$, isothermal at $130^{\circ} \mathrm{C}$ for $2 \mathrm{~min}$, and fourth ramp of $20^{\circ} \mathrm{C} / \mathrm{min}$ up to $240^{\circ} \mathrm{C}$. The transfer line was set at $250^{\circ} \mathrm{C}$. Internal standard was 3-octanol. The MS was performed in a PolarisQ ion trap machine (Thermo Finnigan, Villebon Sur Yvette, France) with a source temperature of $200{ }^{\circ} \mathrm{C}$, ionization of $70 \mathrm{eV}$, and the multiplier offset was 0 volts.

\subsection{Statistical Analyses}

Statistical analysis was performed using the Analyze-it software for Microsoft Excel (version 2.20) and the JMP routine of the SAS software for ANOVA analysis. 


\section{Results}

\subsection{Sampling and Yeast Identification}

Yeasts used on this work, presented on Table 1, were isolated from a very rustic mezcal production winery, from freshly pressed cooked agave must to the final stage of fermentation, spanning in total a ten-day process. Due to the working conditions on the winery, it was possible to sample only at pressing and early fermentation stages, when the vats are being fed fresh agave must during a couple of days, and at end of the fermentation, as the producer covers the fermentation vats with straw and mud, and it is not possible to open them mid-process. Nonetheless, we found a very high yeast diversity in these samples. Strains used represent the productive diversity of this fermentation.

As it can be noticed on Table 1, S. cerevisiae strains were found typically at the end of the fermentation, as expected, while non-Saccharomyces strains were isolated at the pressing and early fermentation stages.

\subsection{Fermentation Performance of the Mezcal Yeast Strains in Semi-Synthetic Medium M3}

On medium M3, the two hexoses were rapidly consumed during the first $24 \mathrm{~h}$, but, by $72 \mathrm{~h}$ of fermentation, less fructose was consumed as compared to glucose, as previously reported [8]. Control strain Fermichamp completed the fermentation after $96 \mathrm{~h}$ of inoculation. Hence, for this first part of screening, the fermentations of all the 24 tested strains were sampled at this time, to compare their productivity (Table 2) and volatile metabolite profile (Table 3).

Table 2. Primary metabolite profiles and hexose consumption for all the yeasts tested at $96 \mathrm{~h}$ of fermentation in synthetic medium M3, $200 \mathrm{~g} / \mathrm{L}$ of initial sugars.

\begin{tabular}{|c|c|c|c|c|c|c|c|}
\hline \multirow{2}{*}{ Strain } & Glucose & Fructose & Ethanol & $\mathrm{CO}_{2}$ & Glycerol & $\begin{array}{l}\text { Acetic } \\
\text { Acid }\end{array}$ & $\begin{array}{c}\text { Dry } \\
\text { Weight }\end{array}$ \\
\hline & \multicolumn{7}{|c|}{$(\mathrm{g} / \mathrm{L})$} \\
\hline Fermichamp & $0.0 \pm 0.0$ & $4.7 \pm 1.7$ & $67.2 \pm 1.4$ & $97.7 \pm 5.7$ & $2.6 \pm 0.1$ & $0.5 \pm 0.1$ & $6.0 \pm 0.3$ \\
\hline $\mathrm{Sc} 3 \mathrm{Y} 2$ & $29.9 \pm 3.9$ & $54.8 \pm 3.1$ & $38.0 \pm 0.2$ & $49.8 \pm 2.0$ & $3.3 \pm 0.2$ & $0.0 \pm 0.0$ & $7.0 \pm 1.7$ \\
\hline Sc3Y3 & $9.9 \pm 1.4$ & $21.7 \pm 2.3$ & $58.8 \pm 13.5$ & $75.8 \pm 19.2$ & $7.3 \pm 1.0$ & $0.8 \pm 0.1$ & $4.9 \pm 0.5$ \\
\hline Sc3Y4 & $3.9 \pm 1.4$ & $13.5 \pm 2.3$ & $61.4 \pm 4.7$ & $83.0 \pm 11.4$ & 2. $7 \pm 0.1$ & $0.5 \pm 0.03$ & $3.9 \pm 0.4$ \\
\hline Sc3Y5 & $9.9 \pm 6.7$ & $26.9 \pm 9.7$ & $57.7 \pm 7.3$ & $72.2 \pm 11.6$ & $6.7 \pm 0.1$ & $0.5 \pm 0.3$ & $4.5 \pm 0.3$ \\
\hline Sc3Y8 & $9.9 \pm 1.5$ & $22.1 \pm 1.3$ & $53.3 \pm 5.8$ & $84.5 \pm 4.36$ & $2.6 \pm 0.2$ & $0.6 \pm 0.2$ & $3.8 \pm 0.2$ \\
\hline Scmosca3 & $0.0 \pm 0.0$ & $2.7 \pm 0.5$ & $67.9 \pm 0.4$ & $94.0 \pm 0.46$ & $6.9 \pm 0.2$ & $0.3 \pm 0.1$ & $5.7 \pm 0.2$ \\
\hline Sc3D6 & $0.3 \pm 0.5$ & $6.6 \pm 2.7$ & $68.4 \pm 7.1$ & $90.7 \pm 1.42$ & $8.2 \pm 0.5$ & $0.5 \pm 0.03$ & $5.9 \pm 0.2$ \\
\hline Sc3D5 & $1.1 \pm 1.6$ & $9.0 \pm 10.0$ & $65.1 \pm 4.9$ & $90.0 \pm 8.0$ & $6.3 \pm 0.2$ & $0.3 \pm 0.1$ & $5.7 \pm 0.1$ \\
\hline Sc3D4 & $0.4 \pm 0.6$ & $6.5 \pm 5.6$ & $66.0 \pm 2.8$ & $89.4 \pm 7.3$ & $6.9 \pm 0.3$ & $0.5 \pm 0.03$ & $5.4 \pm 0.2$ \\
\hline Sc3D2 & $1.2 \pm 0.3$ & $12.5 \pm 1.7$ & $65.4 \pm 1.2$ & $88.3 \pm 2.0$ & $8.3 \pm 0.1$ & $0.9 \pm 0.01$ & $5.6 \pm 0.1$ \\
\hline Sc4Y3 & $0.0 \pm 0.0$ & $3.6 \pm 2.9$ & $67.2 \pm 0.2$ & $92.9 \pm 0.6$ & $7.4 \pm 0.1$ & $0.3 \pm 0.01$ & $5.7 \pm 0.1$ \\
\hline Km4D3 & $30.2 \pm 24.2$ & $51.6 \pm 17.8$ & $34.5 \pm 17.2$ & $53.6 \pm 24.2$ & $5.3 \pm 2.39$ & $0.5 \pm 0.5$ & $4.8 \pm 0.2$ \\
\hline Km1D5 & $33.2 \pm 0.5$ & $57.2 \pm 3.9$ & $29.8 \pm 2.1$ & $46.0 \pm 2.5$ & $5.8 \pm 0.67$ & $0.2 \pm 0.2$ & $6.6 \pm 0.6$ \\
\hline Km1Y9 & $12.2 \pm 12.9$ & $34.8 \pm 16.6$ & $45.3 \pm 17.2$ & $66.5 \pm 19.4$ & $6.2 \pm 1.90$ & $0.5 \pm 0.3$ & $5.1 \pm 0.6$ \\
\hline Td1AN9 & $7.1 \pm 5.2$ & $27.8 \pm 10.3$ & $50.6 \pm 7.6$ & $77.9 \pm 6.5$ & $6.6 \pm 0.7$ & $0.9 \pm 0.2$ & $4.5 \pm 0.5$ \\
\hline Td1AN2 & $59.1 \pm 3.0$ & $67.9 \pm 4.7$ & $20.0 \pm 3.1$ & $27.4 \pm 4.4$ & $3.8 \pm 0.4$ & $0.0 \pm 0.0$ & 6. $9 \pm 0.8$ \\
\hline Td1AN1 & $35.2 \pm 3.9$ & $58.3 \pm 4.5$ & $31.2 \pm 0.2$ & $37.4 \pm 2.6$ & $2.9 \pm 0.03$ & $0.0 \pm 0.0$ & $8.2 \pm 0.4$ \\
\hline Pk4D6 & $37.0 \pm 4.8$ & $60.8 \pm 7.7$ & $25.2 \pm 2.9$ & $34.7 \pm 0.5$ & $5.4 \pm 0.7$ & $0.2 \pm 0.03$ & $6.15 \pm 0.5$ \\
\hline Pm1AN3 & $25.9 \pm 4.9$ & $51.4 \pm 3.6$ & $39.7 \pm 3.7$ & $49.6 \pm 6.5$ & $2.8 \pm 0.2$ & $0.0 \pm 0.0$ & $5.86 \pm 0.7$ \\
\hline Pg1Y12 & $83.0 \pm 0.4$ & $83.0 \pm 0.4$ & $6.3 \pm 0.2$ & $6.7 \pm 1.3$ & $0.4 \pm 0.04$ & $0.0 \pm 0.0$ & $7.4 \pm 1.0$ \\
\hline $\mathrm{Cl} 4 \mathrm{Y} 4$ & $24.8 \pm 12.4$ & $48.6 \pm 10.8$ & $40.0 \pm 7.0$ & $49.3 \pm 11.7$ & $3.03 \pm 0.5$ & $0.0 \pm 0.0$ & $6.2 \pm 1.0$ \\
\hline Cp1Y7 & $45.8 \pm 0.2$ & $64.0 \pm 0.3$ & $19 \pm 0.01$ & $28.6 \pm 5.1$ & $5.7 \pm 0.2$ & $0.2 \pm 0.0$ & $7.8 \pm 1.4$ \\
\hline $\mathrm{RmP12}$ & $70.8 \pm 19.6$ & $76.2 \pm 7.6$ & $11.6 \pm 13.9$ & $13.3 \pm 9.9$ & $1.4 \pm 1.9$ & $0.5 \pm 0.03$ & $5.0 \pm 1.3$ \\
\hline Zb3Y1 & $46.2 \pm 17.7$ & $0.64 \pm 0.3$ & $56.3 \pm 2.0$ & $66.6 \pm 10.0$ & $8.4 \pm 0.1$ & $0.3 \pm 0.02$ & $6.4 \pm 0.6$ \\
\hline
\end{tabular}

All data are the average of three different minibioreactor samples taken by duplicate, and standard deviation is reported. 
Table 3. Volatile profiles of all mezcal yeast tested at $96 \mathrm{~h}$ of fermentation in synthetic medium M3, $200 \mathrm{~g} / \mathrm{L}$ of initial sugars.

\begin{tabular}{|c|c|c|c|c|c|c|c|}
\hline \multirow[t]{2}{*}{ Strain } & $\begin{array}{l}\text { Isoamyl } \\
\text { Alcohol }\end{array}$ & $\begin{array}{l}\text { Isoamyl } \\
\text { Acetate }\end{array}$ & $\begin{array}{l}\text { Phenyl } \\
\text { Ethyl } \\
\text { Acetate }\end{array}$ & $\begin{array}{c}\text { Ethyl } \\
\text { Decanoate }\end{array}$ & $\begin{array}{c}\text { Ethyl } \\
\text { Octanoate }\end{array}$ & $\begin{array}{c}\text { Ethyl } \\
\text { Hexanoate }\end{array}$ & $\begin{array}{c}\text { Ethyl } \\
\text { Butyrate }\end{array}$ \\
\hline & $(\mathrm{mg} / \mathrm{L})$ & \multicolumn{6}{|c|}{$(\mu \mathrm{g} / \mathrm{L})$} \\
\hline Fermichamp & $74.0 \pm 4.2$ & $43.0 \pm 2.8$ & $76.5 \pm 23.3$ & $13.0 \pm 5.0$ & $49.5 \pm 17.7$ & $59.0 \pm 8.5$ & ND \\
\hline $\mathrm{Sc} 3 \mathrm{Y} 2$ & $47.5 \pm 4.9$ & D & $12.5 \pm 4.95$ & $\mathrm{D}$ & D & ND & ND \\
\hline Sc3Y3 & $62.5 \pm 24.7$ & $25.5 \pm 19.1$ & $28.0 \pm 1.4$ & $7.5 \pm 2.1$ & $31.0 \pm 1.4$ & $53.5 \pm 14.9$ & ND \\
\hline Sc3Y4 & $66.0 \pm 1.4$ & $36.0 \pm 2.8$ & $49.0 \pm 4.2$ & $21.0 \pm 1.7$ & $58.0 \pm 0.01$ & $73.5 \pm 6.4$ & ND \\
\hline Sc3Y5 & $52.5 \pm 12.0$ & $25.0 \pm 14.1$ & $123 \pm 5.0$ & $8.5 \pm 0.7$ & $15 \pm 7.1$ & $48.5 \pm 16.3$ & ND \\
\hline Sc3Y8 & $49.0 \pm 4.2$ & D & $16.0 \pm 1.4$ & D & D & ND & ND \\
\hline Scmosca3 & $59.5 \pm 21.9$ & $28.0 \pm 0.0$ & $187 \pm 40.0$ & $7.5 \pm 5.0$ & $77.0 \pm 8.0$ & $76.0 \pm 9.0$ & ND/D \\
\hline Sc3D6 & $92.0 \pm 7.1$ & $42.0 \pm 1.4$ & $32.0 \pm 12.7$ & $9.0 \pm 2.8$ & $35.5 \pm 12.0$ & $69.0 \pm 7.1$ & $215 \pm 8$ \\
\hline Sc3D5 & $78.0 \pm 8.5$ & $27.0 \pm 1.4$ & $35.5 \pm 12.0$ & $12.0 \pm 4.2$ & $83.0 \pm 14.1$ & $82.0 \pm 8.5$ & $205 \pm 8$ \\
\hline Sc3D4 & $112 \pm 0.7$ & $46.5 \pm 2.1$ & $42.5 \pm 3.5$ & $15.5 \pm 5.0$ & $74.5 \pm 19.1$ & $81.0 \pm 8.5$ & 0.0 \\
\hline Sc3D2 & $67.5 \pm 9.2$ & $32.0 \pm 2.8$ & $47.0 \pm 9.9$ & $10.0 \pm 0.01$ & $48.0 \pm 1.4$ & $55.0 \pm 4.2$ & 0.0 \\
\hline Sc4Y3 & $74.0 \pm 1.4$ & $30.5 \pm 0.7$ & $25.5 \pm 16.3$ & $13.5 \pm 6.4$ & $71.0 \pm 18.4$ & $85.5 \pm 0.7$ & $230 \pm 8$ \\
\hline $\mathrm{Km} 4 \mathrm{D} 3$ & $60.5 \pm 27.6$ & $33 \pm 0.0$ & $1693 \pm 574$ & $\mathrm{D}$ & $2.0 \pm 0.01$ & ND & ND \\
\hline Km1D5 & $15.0 \pm 1.4$ & $1774 \pm 588$ & $2422 \pm 186$ & $\mathrm{D}$ & $5.5 \pm 0.7$ & ND & ND \\
\hline Km1Y9 & $79.5 \pm 23.3$ & $23.5 \pm 19.0$ & $2772 \pm 743$ & $\mathrm{D}$ & $2.0 \pm 0.01$ & ND & ND \\
\hline Td1AN9 & $75.5 \pm 9.2$ & $13.5 \pm 0.7$ & $2594 \pm 395$ & $\mathrm{D}$ & D & ND & ND \\
\hline Td1AN2 & $44.0 \pm 21.2$ & ND & $100 \pm 13$ & $\mathrm{D}$ & $3.0 \pm 0.01$ & ND & ND \\
\hline Td1AN1 & $31.0 \pm 4.2$ & ND & $5.0 \pm 1.0$ & $\mathrm{D}$ & $\mathrm{D}$ & ND & ND \\
\hline Pk4D6 & $11.0 \pm 0.0$ & $414 \pm 136$ & $4754 \pm 821$ & $\mathrm{D}$ & $2.0 \pm 0.01$ & ND & ND \\
\hline Pm1AN3 & $41.0 \pm 5.7$ & ND & $19.5 \pm 3.5$ & $\mathrm{D}$ & D & ND & ND \\
\hline Pg1Y12 & $15.5 \pm 0.7$ & ND & $1.0 \pm 0.01$ & $\mathrm{D}$ & $\mathrm{D}$ & ND & ND \\
\hline Cp1Y7 & ND & $353 \pm 64$ & $5211 \pm 452$ & $\mathrm{D}$ & $2.0 \pm 0.01$ & ND & ND \\
\hline $\mathrm{Cl} 4 \mathrm{Y} 4$ & $45.5 \pm 5.0$ & ND & $13.0 \pm 6.0$ & $\mathrm{D}$ & $\mathrm{D}$ & ND & ND \\
\hline $\mathrm{RmP12}$ & $21.0 \pm 0.0$ & ND & \pm 9.8 & $\mathrm{D}$ & $3.0 \pm 0.01$ & ND & ND \\
\hline Zb3Y1 & $61.0 \pm 2.8$ & $12.0 \pm 4.2$ & $143 \pm 36.1$ & $\mathrm{D}$ & $\mathrm{D}$ & ND & ND \\
\hline
\end{tabular}

ND: Not detected; D: concentration below the lower limit of the calibration curve. All data are the average of three different minibioreactor samples, and standard deviation is reported.

As can be seen in Table 2, all the yeast species were able to produce ethanol, with S. cerevisiae strains being the most productive, as expected, but some of the non-Saccharomyces strains were also able to produce above $45 \mathrm{~g} / \mathrm{L}$ (Km1Y9, Td1AN9 and Zb3Y1) during this fermentation time. Regarding residual sugars, it is worth noting that, at the 96-h sampling time, fermentations with the S. cerevisiae 3D series had lower residual sugar concentrations, but, at the end of fermentation $(360 \mathrm{~h}$, data not shown), the fermentations with the 3Y series (except Sc3Y2, which was a high glycerol producer) had almost completely consume both sugars.

Besides some S. cerevisiae strains (Sc3Y3, Sc3Y4, Sc4Y3, Scmosca3 and Fermichamp), only strain Zb3Y1 was able to consume fructose almost completely (residual fructose below $2 \mathrm{~g} / \mathrm{L}$ ). However, it did not consume glucose completely (residual of $8.5 \mathrm{~g} / \mathrm{L}$ ) by the end of the fermentations ( $360 \mathrm{~h}$, data not shown), and it also produced a high amount of glycerol as compared with the other non-Saccharomyces strains. The non-Saccharomyces strains, although less productive in terms of ethanol, were very interesting from the point of view that acetic acid was not produced, or it was but in very low quantities.

\subsection{Volatile Productions of the Mezcal Yeast Strains in Medium M3}

Concerning volatile metabolites produced on the semi-synthetic medium M3, marked differences were observed (Table 3 ) in the production levels of all volatile compounds tested in this work, even among strains of the same species, as observed for the S. cerevisiae group.

Most of the $S$. cerevisiae strains produced ethyl hexanoate, but this volatile was not detected in any of the non-Saccharomyces strains. Among all the strains (Saccharomyces and non-Saccharomyces) only three (Sc3D5, Sc3D6, and Sc4Y3) produced ethyl butyrate. Neither hexyl acetate nor 1-pentanol was 
detected for any of the yeast strains under the conditions tested. Concerning phenyl ethyl acetate, it was produced in high amounts by the three strains belonging to K. marxianus (Km4D3, Km1D5, and Km1Y9) and also by strains Td1AN9 (T. delbrueckii), Pk4D6 (Pichia kluyveri), and Cp1Y7 (Clavispora lusitaniae). For the next stage on grape juice, we tested strains Saccharomyces and non-Saccharomyces individually and in mixed culture (co-culture). The three chosen Saccharomyces cerevisiae strains (Sc3Y3, Sc3Y4, and Sc3Y8, plus control strain Fermichamp) were previously selected based on their global stress tolerance [6]. These mezcal strains belong to tolerance group 1, meaning a high tolerance to ethanol, with or without a hexose present and also tolerant to an initial fructose concentration of $500 \mathrm{~g} / \mathrm{L}$. Nonnon-Saccharomyces strains were chosen based on their high ethanol production (above $45 \mathrm{~g} / \mathrm{L}$, Table 2) and relative fructophilic. According to this, strains Km1Y9, Td1AN9 were selected, and strain Zb3Y1 was also included due to its high ethanol production and for being the most fructophilic (Table 2) of all strains at the sampled time.

\subsection{Fermentation Performance and Volatile Production of the Selected Yeasts, Individually and as Mixed Inoculum in Grape Juice Medium}

Based on their carbon dioxide profiles, we choose a fixed time of $144 \mathrm{~h}$ of fermentation to compare the performance of all strains in grape juice medium, both in individually inoculated (Figure 1 and Table 4) and in mixed cultures (Saccharomyces/non-Saccharomyces, Table 5). From Figure 2, it is clear that strain Zb3Y1 is the best fructose consumer, but it leaves a high amount of glucose in the medium.

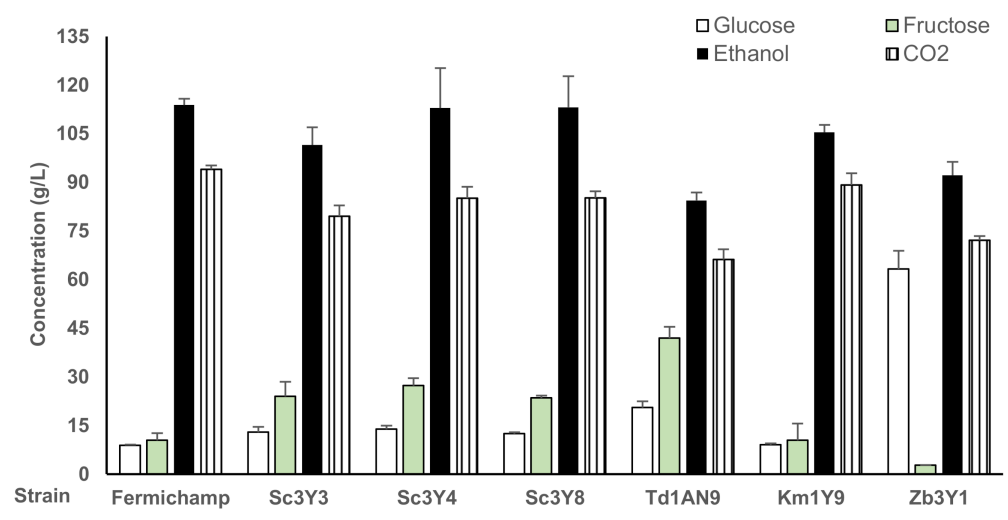

Figure 1. Hexose consumption and primary metabolite production for the selected Saccharomyces and non-Saccharomyces yeasts fermenting individually in grape juice medium at a sugar concentration of $137 \mathrm{~g} / \mathrm{L}$ initial glucose and $119 \mathrm{~g} / \mathrm{L}$ initial fructose at $144 \mathrm{~h}$. Values are the average of six measurements, and standard deviations are presented as error bars.

Table 4. Biomass, acetic acid, and glycerol productions and productivity parameters for the selected Saccharomyces and non-Saccharomyces yeasts fermenting individually in grape juice medium at a sugar concentration of $137 \mathrm{~g} / \mathrm{L}$ initial glucose and $119 \mathrm{~g} / \mathrm{L}$ initial fructose, at $144 \mathrm{~h}$.

\begin{tabular}{ccccccc}
\hline \multirow{2}{*}{ Strain } & Dry Weight & Acetic Acid & Glycerol & $\begin{array}{c}\text { Fermentation } \\
\text { Power (FP) }\end{array}$ & $\begin{array}{c}\text { Fermentation } \\
\text { Purity }\end{array}$ & \multirow{2}{*}{$\mathbf{Y}_{\text {EtOH/S }}$} \\
\cline { 2 - 5 } & \multicolumn{3}{c}{$\mathbf{g} / \mathbf{L}$} & $\mathbf{\%}$ v/v & gacet.ac/FP & \\
\hline Fermichamp & $9.0 \pm 0.3$ & $0.2 \pm 0.0$ & $9.1 \pm 0.2$ & 14.4 & 0.014 & 0.48 \\
Sc3Y3 & $6.2 \pm 0.7$ & $0.7 \pm 0.2$ & $10.2 \pm 1.1$ & 12.9 & 0.054 & 0.46 \\
Sc3Y4 & $6.0 \pm 0.2$ & $0.8 \pm 0.1$ & $10.0 \pm 0.9$ & 14.3 & 0.056 & 0.53 \\
Sc3Y8 & $6.9 \pm 0.4$ & $0.8 \pm 0.1$ & $10.0 \pm 0.8$ & 14.3 & 0.056 & 0.51 \\
Td1AN9 & $4.7 \pm 0.2$ & $0.5 \pm 0.0$ & $8.7 \pm 0.4$ & 10.7 & 0.047 & 0.44 \\
Km1Y9 & $3.6 \pm 0.3$ & $0.7 \pm 0.0$ & $9.9 \pm 0.0$ & 13.4 & 0.052 & 0.45 \\
Zb3Y1 & $6.3 \pm 0.3$ & $0.3 \pm 0.0$ & $7.8 \pm 0.5$ & 11.7 & 0.026 & 0.49 \\
\hline
\end{tabular}

All data are the average of three different minibioreactor samples taken by duplicate, and standard deviation is reported. Fermentation power, purity, and ethanol yields are calculated using metabolites' average values. 
Table 5. Sugar consumption and primary metabolite production for the selected yeasts, fermenting individually, or as mixed inoculum in grape juice medium $(103 \mathrm{~g} / \mathrm{L}$ initial glucose and $105 \mathrm{~g} / \mathrm{L}$ initial fructose), at $144 \mathrm{~h}$.

\begin{tabular}{cccccccc}
\hline \multirow{2}{*}{$\begin{array}{c}\text { Individual or Mixed } \\
\text { Inocula Sc/non-Sc (1:9) }\end{array}$} & Glucose & Fructose & Ethanol & $\mathbf{C O}_{2}$ & Glycerol & Ac. Acid & $\begin{array}{c}\text { Dry } \\
\text { Weight }\end{array}$ \\
\cline { 2 - 7 } & & & & & & \\
\hline Fermichamp & $6.3 \pm 0.09$ & $5.6 \pm 1.2$ & $68.0 \pm 1.9$ & $99.4 \pm 3.0$ & $8.0 \pm 0.2$ & $0.5 \pm 0.1$ & $11.4 \pm 0.2$ \\
Fcham/Td1AN9 & $3.4 \pm 2.6$ & $7.5 \pm 2.7$ & $82.2 \pm 1.1$ & $92.9 \pm 1.6$ & $7.9 \pm 0.3$ & $0.3 \pm 0.1$ & $8.6 \pm 0.4$ \\
Fcham/Km1Y9 & $3.5 \pm 2.7$ & $6.8 \pm 1.7$ & $84.5 \pm 2.3$ & $94.5 \pm 3.9$ & $8.1 \pm 0.2$ & $0.4 \pm 0.1$ & $8.4 \pm 0.3$ \\
Fcham/Zb3Y1 & $3.8 \pm 3.0$ & $7.8 \pm 4.1$ & $82.1 \pm 1.0$ & $91.8 \pm 3.0$ & $4.8 \pm 1.0$ & $1.2 \pm 0.5$ & $8.3 \pm 0.6$ \\
& & & & & & & \\
Sc3Y3 & $7.1 \pm 0.5$ & $14.0 \pm 2.0$ & $66.0 \pm 1.2$ & $98.6 \pm 2.5$ & $8.0 \pm 0.2$ & $0.5 \pm 0.0$ & $7.6 \pm 0.4$ \\
Sc3Y3/Td1AN9 & $9.2 \pm 1.7$ & $24.9 \pm 4.7$ & $75.7 \pm 1.7$ & $86.4 \pm 2.5$ & $8.4 \pm 0.4$ & $1.2 \pm 0.2$ & $5.9 \pm 0.2$ \\
Sc3Y3/Km1Y9 & $11.4 \pm 3.4$ & $31.9 \pm 7.1$ & $73.4 \pm 6.5$ & $77.1 \pm 2.0$ & $7.6 \pm 1.1$ & $1.2 \pm 0.4$ & $5.4 \pm 0.2$ \\
Sc3Y3/Zb3Y1 & $14.4 \pm 6.0$ & $17.9 \pm 6.1$ & $79.1 \pm 3.9$ & $84.4 \pm 5.9$ & $6.3 \pm 2.0$ & $1.2 \pm 0.6$ & $6.5 \pm 0.6$ \\
& & & & & & & \\
Sc3Y4 & $8.1 \pm 1.8$ & $16.4 \pm 5.9$ & $67.0 \pm 3.3$ & $94.7 \pm 5.4$ & $8.4 \pm 0.3$ & $0.6 \pm 0.1$ & $7.5 \pm 0.7$ \\
Sc3Y4/Td1AN9 & $11.3 \pm 2.3$ & $30.0 \pm 4.6$ & $73.3 \pm 1.7$ & $78.9 \pm 3.8$ & $7.5 \pm 0.1$ & $0.5 \pm 0.2$ & $5.6 \pm 0.3$ \\
Sc3Y4/Km1Y9 & $9.2 \pm 3.5$ & $25.0 \pm 8.9$ & $76.4 \pm 3.9$ & $81.3 \pm 4.1$ & $7.1 \pm 0.4$ & $0.8 \pm 0.3$ & $5.1 \pm 0.3$ \\
Sc3Y4/Zb3Y1 & $20.8 \pm 5.0$ & $23.6 \pm 5.1$ & $71.8 \pm 4.0$ & $78.4 \pm 5.8$ & $5.0 \pm 1.2$ & $1.3 \pm 0.8$ & $5.2 \pm 0.3$ \\
Sc3Y8 & $9.5 \pm 2.8$ & $19.5 \pm 7.4$ & $65.8 \pm 2.7$ & $1010 \pm 6.6$ & $7.3 \pm 1.1$ & $0.76 \pm 0.3$ & $7.0 \pm 0.6$ \\
Sc3Y8/Td1AN9 & $15.4 \pm 8.2$ & $35.8 \pm 14.3$ & $70.0 \pm 7.8$ & $76.4 \pm 11.1$ & $7.8 \pm 0.3$ & $0.9 \pm 0.4$ & $5.6 \pm 0.3$ \\
Sc3Y8/Km1Y9 & $9.8 \pm 2.3$ & $27.7 \pm 6.0$ & $75.9 \pm 1.0$ & $80.9 \pm 5.6$ & $7.4 \pm 0.3$ & $1.0 \pm 0.3$ & $5.5 \pm 0.4$ \\
Sc3Y8/Zb3Y1 & $17.3 \pm 5.2$ & $23.0 \pm 4.8$ & $81.6 \pm 7.0$ & $79.6 \pm 5.5$ & $7.4 \pm 1.0$ & $0.7 \pm 0.4$ & $6.2 \pm 0.6$ \\
\hline
\end{tabular}

Values are the average of three different minibioreactor samples taken by duplicate, and standard deviations are reported.

As seen in Table 4, fermentations on grape juice had high yields for all Saccharomyces and non-Saccharomyces strains.

Next, fermentation performances were compared for the mixed inocula experiments to verify the effect of the co-inoculation on productivity for individually fermenting and on mixed inocula fermentation (Table 5), to assess the effect of the yeasts' combinations. It is worth noting that the natural grape juice medium used in the experiments for Tables 4 and 5 were from different juice stocks; hence, the difference in the total sugar residual concentrations at the same sampling time. As reference, values of metabolite productions of the individual S. cerevisiae strains are included again in Table 5 to be compared with their mixed inocula fermentations.

An increase in ethanol production was observed when mixed inocula were used, with respect to the pure S. cerevisiae strain used, but, also, some of the mixtures increased their acetic acid production beyond acceptable quality levels (less than $1 \mathrm{~g} / \mathrm{L}$ ), specifically those including S. cerevisiae Sc3Y3, K. marxianus Km1Y9 or Z. bailii Zb3Y1 strains.

Due to its better overall aroma, lower ethanol production, and low acidity and higher fermentation purity, we decided to analyze the non-Saccharomyces strain Td1AN9in more detail. The kinetic behavior for primary metabolites (Figure 2) was assessed on mixed cultures with different S. cerevisiae strains having in common the Td1AN9 strain as the non-Saccharomyces couple. As it can be seen in Figure 2 for the whole fermentation profile for the mixed cultures using Td1AN9 as the non-Saccharomyces strain, there was a fast sugar consumption for the three mixed cultures tested, and maximal ethanol production was obtained between 120 and $144 \mathrm{~h}$ of fermentation. 


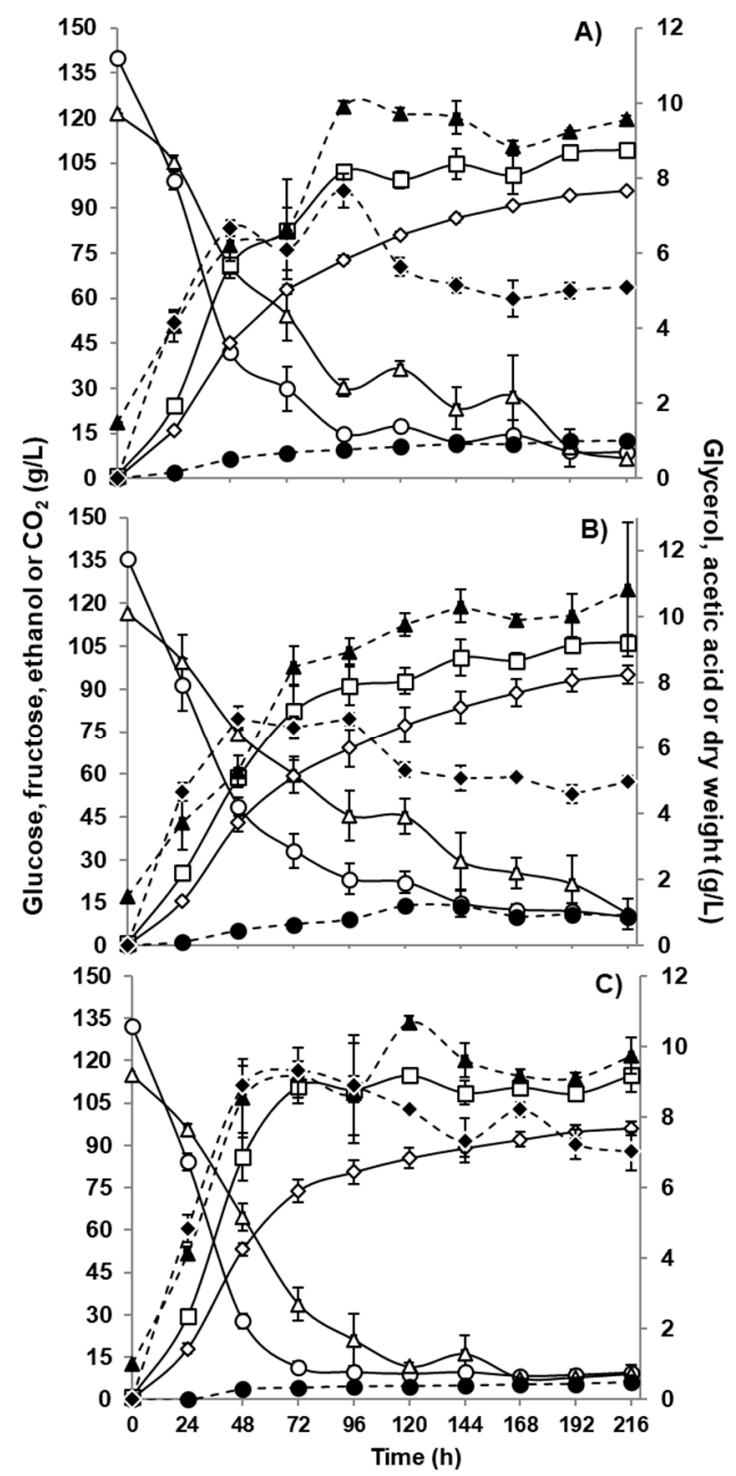

Figure 2. Fermentation kinetics on grape juice medium for the three Saccharomyces cerevisiae/Torulaspora delbrueckii (Td1AN9) mixed inoculum tested: (A) Sc3Y4/Td1AN9, (B) Sc3Y8/Td1AN9, and (C) Fermichamp/Td1AN9. Markers are average values of: (-○-) Glucose, $(-\triangle-)$ fructose, $(-\square-)$

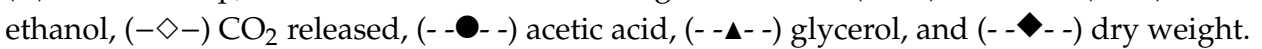

Growth characterized as global values of optical densities and viability showed that, mixed culture containing the control strain Fermichamp grew faster (Figure 3A), and this was due to a higher percentage of such S. cerevisiae strain thriving on the grape juice (Figure 3B). Although total population remained high (no lysed cells were observed under the microscope, without methylene blue), the viability rapidly declined after $72 \mathrm{~h}$ of culture when assessed by methylene blue staining. It is worth noticing that viability of $T$. delbrueckii at $144 \mathrm{~h}$ of grape juice fermentations was higher when combined with mezcal yeasts than with the control strain Fermichamp (Figure 3B, full markers), even considering that all three fermentations had the same amount of ethanol, around $100 \mathrm{~g} / \mathrm{L}$. 


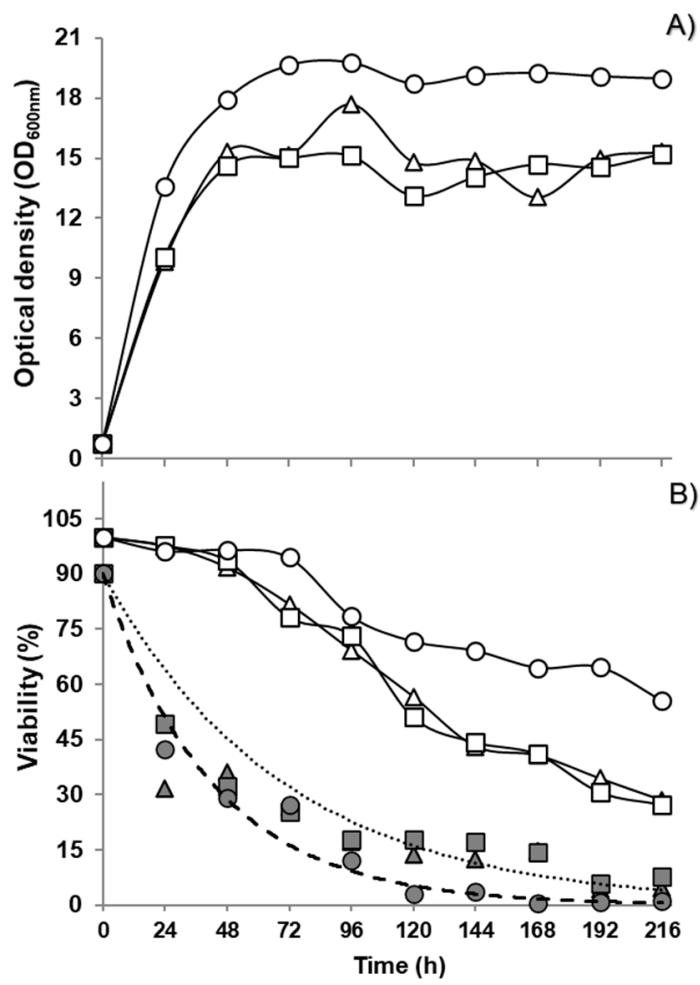

Figure 3. Culture growth on grape juice medium for the three Saccharomyces cerevisiae/Torulaspora delbrueckii mixed inoculum fermentations tested, expressed as: (A) Optical density at $600 \mathrm{~nm},(-\Delta-)$ Sc3Y4/Td1AN9, (-口-) Sc3Y8/Td1AN9; (-O-) Fermichamp/Td1AN9; and (B) Percentage of viability of the whole culture (open markers, determined by methylene blue staining) and that of Torulaspora delbrueckii as determined on WL Differential and WL Nutrient agar media, (…....) Sc3Y4/Td1AN9, (- - - - - -) Sc3Y8/Td1AN9; (- - - - - ) Fermichamp/Td1AN9. Exponential decay fittings shown with dotted and slashed lines. Standard deviations were always less than $10 \%$; hence, the deviation bars are omitted in the graphs for clarity.

Population kinetics analysis (Figure 4) of the mixed inoculum fermentations showed that, for the first $24 \mathrm{~h}$ of fermentation the two populations grew rapidly and maintained a high population from 24 to $48 \mathrm{~h}$ of fermentation. The $S$. cerevisiae strains in general maintained their population at around $5 \times 10^{7}$ cells $/ \mathrm{mL}$ until the end of the culture, whilst the population of the non-Saccharomyces strain Td1AN9 declined by around $90 \%$ (between 1 to $5 \times 10^{6}$ cell $/ \mathrm{mL}$ ). This strain was most affected by control strain Fermichamp (Figure 4C), but never completely disappearing. At the end of fermentation, the whole viability (Saccharomyces and non-Saccharomyces) of the culture remained at values around $50 \%$ as shown in Figure 3B. This meant that viable yeast population was comprised mainly by the S. cerevisiae strain inoculated, but, still, the non-Saccharomyces strains was present and metabolically active in high quantities.

Finally, volatile compounds produced by the mixed inocula fermentations in grape juice medium were strongly influenced by the two strains used (Table 6). The presence of the S. cerevisiae strains determined, mainly, the isoamyl alcohol, ethyl octanoate, and ethyl decanoate concentrations, whilst T. delbrueckii strain 1AN9 determined most of the phenyl ethyl acetate concentrations. For the ethyl hexanoate, it was the average contribution of both strains. Although Td1AN9 is capable, as individual inoculum, to produce isoamyl acetate, only its combination with control strain Fermichamp (which also produces isoamyl acetates individually) produced this compound, but its production by Td1AN9 was inhibited when mixed with the S. cerevisiae strains from mezcal. For the mixed culture with Sc3Y4 and Sc3Y8, isoamyl acetate concentrations were present but below the detection limit. In general terms, when using mixed inocula, we observed an increase on isoamyl alcohol levels as compared to pure 
inoculum of S. cerevisiae strains, as well as a decrease in phenyl ethyl acetate concentration in the fermented product, as compared to the use of the non-Saccharomyces strain as pure inoculum.

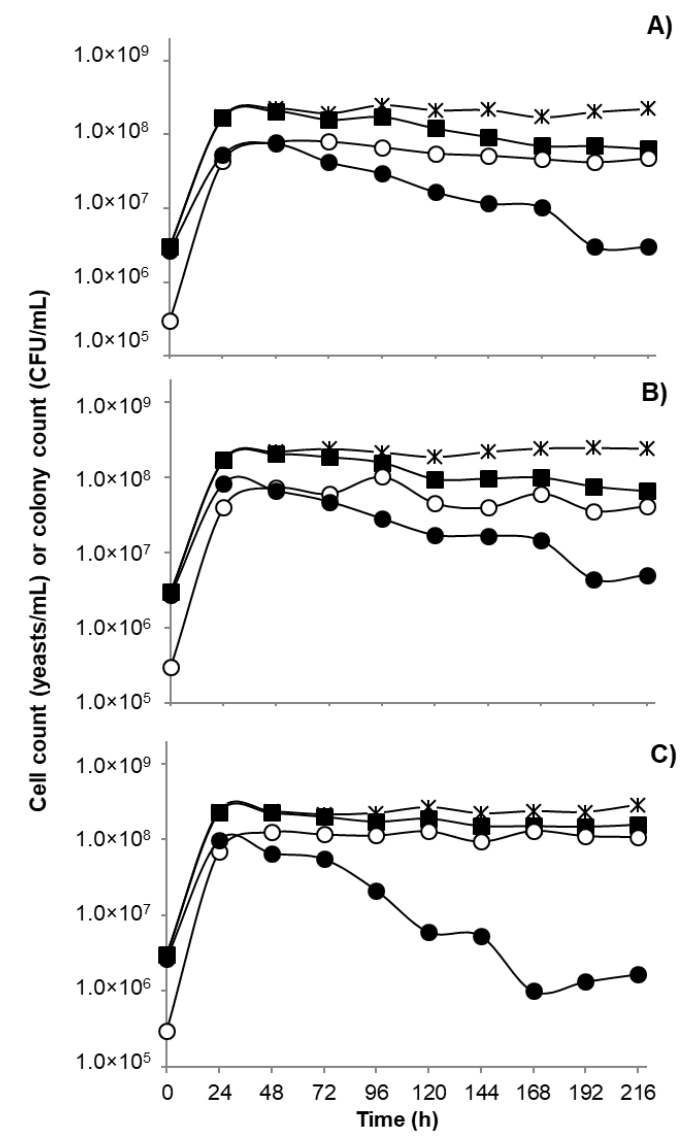

Figure 4. Population kinetics on grape juice medium for the three Saccharomyces cerevisiae/Torulaspora delbrueckii mixed inoculum tested: (A) Sc3Y4/Td1AN9, (B) Sc3Y8/Td1AN9 and (C) Fermichamp/Td1AN9; Markers show: (-K-) total cells count, (-口-) viable cell count, both determined by a Neubauer chamber without or with methylene blue, respectively; (-O-) S. cerevisiae colonies count, and (-•-) T. delbrueckii colonies count, determined by solid (WL Differential and WL Nutrient) media. As data is presented in logarithmic scale, standard deviation bars are masked by marker size.

Table 6. Main volatile compounds profiles of the selected Saccharomyces and non-Saccharomyces yeasts fermenting individually (upper panel) or as a mixed culture (bottom panel) in grape juice medium at a sugar concentration of $137 \mathrm{~g} / \mathrm{L}$ initial glucose and $119 \mathrm{~g} / \mathrm{L}$ initial fructose, data at $144 \mathrm{~h}$ of culture.

\begin{tabular}{|c|c|c|c|c|c|c|}
\hline \multirow{2}{*}{ Strain } & $\begin{array}{l}\text { Isoamyl } \\
\text { Alcohol }\end{array}$ & $\begin{array}{l}\text { Isoamyl } \\
\text { Acetate }\end{array}$ & $\begin{array}{l}\text { Phenyl Ethyl } \\
\text { Acetate }\end{array}$ & $\begin{array}{c}\text { Ethyl } \\
\text { Decanoate }\end{array}$ & $\begin{array}{c}\text { Ethyl } \\
\text { Octanoate }\end{array}$ & $\begin{array}{c}\text { Ethyl } \\
\text { Hexanoate }\end{array}$ \\
\hline & $(\mathrm{mg} / \mathrm{L})$ & \multicolumn{5}{|c|}{$(\mu \mathrm{g} / \mathrm{L})$} \\
\hline Fermichamp & $174 \pm 5.0$ & $111 \pm 9.2$ & $133 \pm 14.1$ & $133 \pm 2.1$ & $61 \pm 4.9$ & $40 \pm 2.1$ \\
\hline Sc3Y3 & $77 \pm 4.5$ & D & $70 \pm 16.5$ & $140 \pm 5.3$ & $64 \pm 4.2$ & $38 \pm 3.5$ \\
\hline Sc3Y4 & $78 \pm 0.6$ & $\mathrm{D}$ & $63 \pm 5.7$ & $103 \pm 9.3$ & $63 \pm 2.1$ & $33 \pm 2.3$ \\
\hline $\mathrm{Sc} 3 \mathrm{Y} 8$ & $82 \pm 4.0$ & D & $82 \pm 20.5$ & $115 \pm 27.6$ & $63 \pm 2.7$ & $45 \pm 10.4$ \\
\hline Km1Y9 & $131 \pm 6.0$ & $30 \pm 11.2$ & $5069 \pm 291.2$ & $39 \pm 3.6$ & $38 \pm 1.0$ & D \\
\hline Td1AN9 & $143 \pm 90$ & $45 \pm 14.2$ & $3349 \pm 166.8$ & $32 \pm 0.6$ & $\mathrm{D}$ & $16 \pm 1.5$ \\
\hline Zb3Y1 & $213 \pm 200$ & $\mathrm{D}$ & $139 \pm 9.1$ & $32 \pm 2.1$ & $34 \pm 0.0$ & $15 \pm 0.0$ \\
\hline Fcham/Td1AN9 & $141 \pm 18.8$ & $55 \pm 9.9$ & $543 \pm 92.4$ & $105 \pm 16.5$ & $41 \pm 8.3$ & $23 \pm 3.5$ \\
\hline Sc3Y4/Td1AN9 & $93 \pm 3.2$ & D & $475 \pm 34.9$ & $84 \pm 11.5$ & $37 \pm 8.5$ & $33 \pm 7.0$ \\
\hline Sc3Y8/Td1AN9 & $85 \pm 9.1$ & $\mathrm{D}$ & $544 \pm 8.0$ & $102 \pm 16.5$ & $33 \pm 4.0$ & $26 \pm 2.3$ \\
\hline
\end{tabular}




\section{Discussion}

One of the main objectives of this work was to compare the capabilities of production of aromatic volatile (flavor) compounds of the different mezcal strains when fermenting on a wine-type synthetic medium (M3), and in real grape juice, also, with a single strain inoculum or co-inoculated in a mixed fashion. Semi-synthetic medium M3 allowed us to compare the individual productive behavior of all the 24 strains. We found that ethanol was produced by all the strains, being maximal (but variable amongst strains) for $S$. cerevisiae as expected, but it also was produced in good quantities by some of the non-Saccharomyces strains belonging to Kluyveromyces, Torulaspora, and Zygosaccharomyces genera, which made them candidates to be tested in the grape juice medium as part of mixed inoculum with different S. cerevisiae strains, in terms of their displayed natural tolerance to this alcohol.

Concerning specifically to $S$. cerevisiae strains, Camarasa et al. [11] analyzed in a high glucose $(240 \mathrm{~g} / \mathrm{L})$ synthetic medium the phenotypic variability, including the production of aromatic compounds, of a collection of 72 S. cerevisiae strains obtained from seven different ecological niches: bakery, laboratory, natural isolates (plants and soil), clinical isolates, fermentative processes (beer, sake, palm wine), vineyard, and commercial wine. They observed that the larger differences amongst the strains are in their biomass production and formation of by-products but, interestingly, not in their ethanol production levels, different to what was observed in this work (Table 2), specifically for ethanol. These authors concluded that commercial wine strains are characterized by high biomass concentration and good fermentative performance, low acetate production, and low ethyl butyrate synthesis. More recently, and similar to the work presented here for S. cerevisiae mezcal strains, Franco-Duarte et al. [12] established that, for their 24 S. cerevisiae strains, ethanol and organic acids (in particular acetic acid) concentration explained most of the metabolic differences among strains. The S. cerevisiae strains studied in more detail here produce comparable amounts of ethanol as the commercial strain Fermichamp, and the selected strains also led to high glycerol levels and were able to almost completely consume glucose and fructose during fermentation. In general, primary metabolites were produced in higher amounts in the grape juice medium than in the semi-synthetic medium M3.

For the mixed inocula fermentations, we observed an increased glycerol and acetic acid productions in the mixed cultures as compared with data obtained in pure cultures as reported by Reference [13]. In mixed cultures, the S. cerevisiae strains and T. delbrueckii 1AN9 reached their maximal populations at 24 $\mathrm{h}$, similar to what was also reported by Reference [13]. The comparison between the cell concentrations obtained in both nutrient WL (non-selective) and differential WL (no growth of S. cerevisiae) agar media clearly shows that at the beginning of the fermentation process the majority of the population belongs to the non-Saccharomyces strain, as inoculated in higher amounts, but as time proceeds, S. cerevisiae becomes dominant up to the end of fermentation, similar to that reported by Reference [13]. However, unlike these authors, who report a low percentage $(<1 \%)$ of viability for their non-Saccharomyces strains (C. zemplinina and H. uvarum), our strain Td1AN9 have a viability between 10 and 15\% at the end of fermentation, and it is most affected by co-inoculation with control strain Fermichamp, being not due to ethanol concentration, as it was similar in all inoculum combinations. We observed the same phenomenon of a major inhibition due to the presence of control strain Fermichamp for the K. marxianus and Z. bailli strains (data not shown).

Overall, at the conditions tested in this work, the presence of a $S$. cerevisiae strain reduces the growth capacity of Td1AN9 when it is mixed from $48 \mathrm{~h}$ of culture. At this time, the concentration of ethanol is around $65 \mathrm{~g} / \mathrm{L}$ (Figure 2), which is lower to the maximum ethanol production capability by the pure Td1AN9 inoculum fermentation (Figure 1) and where the cell viable count is the same as the individual S. cerevisiae strains, around $1 \times 10^{8}$ cells $/ \mathrm{mL}$. Hence, we cannot attribute solely to ethanol the inhibition/damaging effect over T. delbrueckii cells at this time, although we know that concentrations above $8 \%$ ethanol are stressing on solid media as previously determined for this strain in YPD [6], and that the $S$. cerevisiae strain is taking advantage of the cellular contents leaked by the non-Saccharomyces strain. This is in contrast to what has been reported for H. guilliermondii [14]. These authors tested not only different ratio of species in the mixed inocula but also aerobic conditions and different media 
and S. cerevisiae strains (data not shown by the authors) and concluded that inhibition and death of their non-Saccharomyces strain was due to some unknown compound present and accumulated in the supernatant of $S$. cerevisiae cultures, different than the killer toxins already reported for $S$. cerevisiae. Similarly, it was reported that the main inhibitory mechanism towards their K. thermotolerans and T. delbrueckii strains was the physical presence of $S$. cerevisiae cells [15]. Our results seem to support this latter explanation, although it is clear that such effect most probably is species-specific, as in the work of Kosel et al. [16]. These authors did not find any effect of the cell-to-cell contact of the commercial S. cerevisiae EC1118 over the growth kinetics of the cultures of Dekkera bruxellensis, which is a spoilage yeast of low growth and a low tolerance to high sugar concentration, as well a weak producer of ethanol. All this indicates that more work is needed to clarify the specific properties of $S$. cerevisiae with T. delbrueckii co-cultures.

Concerning volatile metabolites production, we found that different esters and higher alcohols were produced, and there were differences between pure and mixed cultures, being strain dependent as observed also by Reference [17] and, in general, being higher for the mixed rather than for the individually inoculated fermentations. Pure cultures of $S$. cerevisiae strains showed the highest total ester content, except for phenyl ethyl acetate, as compared to the non-Saccharomyces strain Td1AN9. Maturano et al. [17] obtained, for phenyl ethyl acetate, values from 30 to a maximum of $310 \mu \mathrm{g} / \mathrm{L}$ for the varieties of wine analyzed, while, in the results presented here, the non-Saccharomyces yeasts showed values ranging from 130 to $5069 \mu \mathrm{g} / \mathrm{L}$ and, when used a mixed inocula, values were high, ranging from 450 to $540 \mu \mathrm{g} / \mathrm{L}$, higher than those reported by Reference $[17,18]$. This is a positive feature due to the great importance of this compound for its very pleasant floral aroma. As reported by Reference [1], which also worked in wine-type synthetic medium, the production of certain volatile compounds that influence wine aroma was strain-dependent, and they observed that the concentrations of the measured compounds (except acetic acid) varied significantly in function of the inoculated strain, to the point that it permitted their identification, concluding that the combined use of two or more yeast strains or species is an interesting alternative for improving wine quality [19]. In the work of Kosel et al. [16], the non-contact (cultures separated by a membrane) co-culture of S. cerevisiae with D. bruxellensis in a synthetic wine must resulted in a higher production of aromatic ethyl ester compounds, as compared with the pure cultures of the two yeasts. These authors propose a hybrid computational pheno-metabolomic approach to classify and select those $S$. cerevisiae strains with an increased performance on wine making, correlating this selection with a good growth on cycloheximide, on iprodion, and a temperature of $18{ }^{\circ} \mathrm{C}$, the presence of two homozygous alleles (ScAAT6-256 and ScAAT5-256), and a high production of 2-phenylethyl acetate, ethyl butanoate, ethyl hexanoate, and ethyl octanoate.

The co-inoculation of Td1AN9 with any of the two S. cerevisiae mezcal strains prevented/inhibited the production of isoamyl acetate but not when mixed with control strain Fermichamp, which is also capable of producing it. This may indicate a level of recognition and/or compatibility amongst mezcal strains, Saccharomyces and non-Saccharomyces, as this compound (and isoamyl alcohol) has been recently reported as possessing a wide antimicrobial feature when present in a fermented (sake) beverage [20], but also evidenced here by the relatively higher viability of the Td1AN9 strains at the end of the fermentation, when in presence of the two S. cerevisiae mezcal strains, but not with control, wine strain Fermichamp. Overall, isoamyl alcohol concentrations varied between S. cerevisiae yeasts as reported in other studies in wine [17,18] and other fermented beverages [20].

In winemaking, the use of pure yeast cultures allows a better control of the fermentations; however, it can also reduce the production of some desired metabolites, both from the yeast's metabolism itself and from transformation of precursors present in the grape must. For this purpose, it is increasingly seen more convenient to use different yeast genera and species, which can contribute or influence the chemical composition and the flavor of wines $[4,5,12,13,17,19,21]$. The volatile compounds produced by the strains analyzed in this study are of great aromatic value, especially the production of ethyl hexanoate and ethyl octanoate (apple note), isoamyl acetate (banana note), and phenyl ethyl acetate 
(fruity, floral notes), compounds which could render (in the appropriate amounts) good organoleptic characteristics to a wine.

\section{Conclusions}

The main aim of this work was to assess the technological feasibility of using mixed inoculum of yeasts, originally isolated from mezcal, to be used in wine-type grape juice fermentations. We observed that some of the mezcal yeast strains were competitive in terms of primary metabolites and volatile compounds production. Fermentation performance comparisons in a semi-synthetic medium allowed us to choose those strains, Saccharomyces and non-Saccharomyces, with complementary metabolic characteristics to be tested in a real, wine-type fermentation at a small scale and to propose a mixed inoculum prepared with a S. cerevisiae and a T. delbrueckii mezcal strains to obtain balanced aromatic profiles in a model wine product.

Author Contributions: Conceptualization, C.P.L-C. and P.T; methodology, F.J.D.1.T-G., P.T. and C.P.L-C.; formal analysis, J.A.N-Z and C.P.L-C.; investigation, F.J.D.1.T-G. and C.P.L-C.; resources, C.P.L-C., J.A.N-Z and P.T.; data curation, J.A.N-Z. and C.P.L-C.; writing-Original draft preparation, F.J.D.1.T-G.; writing-Review and editing, J.A.N-Z and C.P.L-C.; visualization, J.A.N-Z. and C.P.L-C.; supervision, C.P.L-C.; project administration, C.P.L-C.; funding acquisition, C.P.L-C., J.A.N-Z and P.T. All authors have read and agreed to the published version of the manuscript.

Funding: This research was funded by Ciencia Básica CONACYT 2013-1-221289 (México) and for the doctoral scholarship granted to F.J. De la Torre-González (CONACyT, México).

Acknowledgments: We acknowledge the technical support of M. Morere for the GC-MS analysis (INP-Toulouse).

Conflicts of Interest: The authors declare no conflict of interest. The funder had no role in the design of the study; in the collection, analyses, or interpretation of data; in the writing of the manuscript, or in the decision to publish the results.

\section{References}

1. Barrajón, N.; Capece, A.; Arévalo-Villena, M.; Briones, A.; Romano, P. Co-inoculation of different Saccharomyces cerevisiae strains and influence on volatile composition of wines. Food Microbiol. 2011, 28, 1080-1086. [CrossRef] [PubMed]

2. Arroyo-López, F.N.; Querol, A.; Barrio, E. Application of a substrate inhibition model to estimate the effect of fructose concentration on the growth of diverse Saccharomyces cerevisiae strains. J. Ind. Microbiol. Biotechnol. 2009, 36, 663-669. [CrossRef] [PubMed]

3. Zuzuarregui, A.; del Olmo, M.L. Analyses of stress resistance under laboratory conditions constitute a suitable criterion for wine yeast selection. Antonie Leeuwenhoek Int. J. Gen. Mol. Microbiol. 2004, 85, 271-280. [CrossRef] [PubMed]

4. García, M.; Greetham, D.; Wimalasena, T.T.; Phister, T.G.; Cabellos, J.M.; Arroyo, T. The phenotypic characterization of yeast strains to stresses inherent to wine fermentation in warm climates. J. Appl. Microbiol. 2016, 121, 215-233. [CrossRef] [PubMed]

5. Belda, I.; Ruiz, J.; Alastruey-Izquierdo, A.; Navascués, E.; Marquina, D.; Santos, A. Unraveling the enzymatic basis of wine "flavorome": A phylo-functional study of wine related yeast species. Front. Microbiol. 2016, 7, 12. [CrossRef]

6. De la Torre-González, F.J.; Narváez-Zapata, J.A.; López-y-López, V.E.; Larralde-Corona, C.P. Ethanol tolerance is decreased by fructose in Saccharomyces and non-Saccharomyces yeasts. LWT Food Sci. Technol. 2016, 67. [CrossRef]

7. Peter, J.; De Chiara, M.; Friedrich, A.; Yue, J.-X.; Pflieger, D.; Bergström, A.; Sigwalt, A.; Barre, B.; Freel, K.; Llored, A.; et al. Genome evolution across 1011 Saccharomyces cerevisiae isolates. Nature 2018, 556, 339-344. [CrossRef]

8. Oliva Hernández, A.A.; Taillandier, P.; Reséndez Pérez, D.; Narváez Zapata, J.A.; Larralde Corona, C.P. The effect of hexose ratios on metabolite production in Saccharomyces cerevisiae strains obtained from the spontaneous fermentation of mezcal. Antonie Leeuwenhoek Int. J. Gen. Mol. Microbiol. 2013, 103, 833-843. [CrossRef] 
9. Vergara-Álvarez, I.; Quiroz-Figueroa, F.; Tamayo-órdónñez, M.C.; Oliva-Hernández, A.A.; Larralde-Corona, C.P.; Narváez-Zapata, J.A. Flocculation and expression of FLO genes of a Saccharomyces cerevisiae mezcal strain with high stress tolerance. Food Technol. Biotechnol. 2019, 57, 544-553. [CrossRef]

10. Yan, G.; Zhang, B.; Joseph, L.; Waterhouse, A.L. Effects of initial oxygenation on chemical and aromatic composition of wine in mixed starters of Hanseniaspora vineae and Saccharomyces cerevisiae. Food Microbiol. 2020, 90, 103460. [CrossRef]

11. Camarasa, C.; Sanchez, I.; Brial, P.; Bigey, F.; Dequin, S. Phenotypic landscape of Saccharomyces cerevisiae during wine fermentation: Evidence for origin-dependent metabolic traits. PLoS ONE 2011, 6, e25147. [CrossRef]

12. Franco-Duarte, R.; Umek, L.; Mendes, I.; Castro, C.C.; Fonseca, N.; Martins, R.; Silva-Ferreira, A.C.; Sampaio, P.; Pais, C.; Schuller, D. New integrative computational approaches unveil the Saccharomyces cerevsiae pheno-metabolomic fermentative profile and allow strain selection for winemaking. Food Chem. 2016, 211, 509-520. [CrossRef] [PubMed]

13. Andorrà, I.; Berradre, M.; Rozès, N.; Mas, A.; Guillamón, J.M.; Esteve-Zarzoso, B. Effect of pure and mixed cultures of the main wine yeast species on grape must fermentations. Eur. Food Res. Technol. 2010, 231, 215-224. [CrossRef]

14. Pérez-Nevado, F.; Albergaria, H.; Hogg, T.; Girio, F. Cellular death of two non-Saccharomyces wine-related yeasts during mixed fermentations with Saccharomyces cerevisiae. Int. J. Food Microbiol. 2006, 108, 336-345. [CrossRef]

15. Nissen, P.; Nielsen, D.; Arneborg, N. Viable Saccharomyces cerevisiae cells at high concentrations cause early growth arrest of non-Saccharomyces yeasts in mixed cultures by a cell - Cell contact-mediated mechanism. Yeast 2003, 20, 331-341. [CrossRef]

16. Kosel, J.; Čadež, N.; Schuller, D.; Carreto, L.; Franco-Duarte, R.; Raspor, P. The influence of Dekkera bruxellensis on the transcriptome of Saccharomyces cerevisiae and on the aromatic profile of synthetic wine must. FEMS Yeast Res. 2017, fox018, 9184-9196. [CrossRef]

17. Maturano, Y.P.; Assof, M.; Fabani, M.P.; Nally, M.C.; Jofré, V.; Rodríguez Assaf, L.A.; Toro, M.E.; Castellanos de Figueroa, L.I.; Vazquez, F. Enzymatic activities produced by mixed Saccharomyces and non-Saccharomyces cultures: Relationship with wine volatile composition. Antonie Leeuwenhoek Int. J. Gen. Mol. Microbiol. 2015, 108, 1239-1256. [CrossRef]

18. Jiang, B.; Zhang, Z. Volatile compounds of young wines from cabernet sauvignon, cabernet gernischet and chardonnay varieties grown in the loess plateau region of China. Molecules 2010, 15, 9184-9196. [CrossRef]

19. Romano, P.; Fiore, C.; Paraggio, M.; Caruso, M.; Capece, A. Function of yeast species and strains in wine flavour. Int. J. Food Microbiol. 2003, 86, 169-180. [CrossRef]

20. Ando, H.; Kurata, A.; Kishimoto, N. Antimicrobial properties and mechanism of volatile isoamyl acetate, a main flavour component of Japanese sake (Ginjo-shu). J. Appl. Microbiol. 2015, 118, 873-880. [CrossRef]

21. Chambers, P.J.; Borneman, A.R.; Varela, C.; Cordente, A.G.; Bellon, J.R.; Tran, T.M.T.; Henschke, P.A.; Curtin, C.D. Ongoing domestication of wine yeast: Past, present and future. Aust. J. Grape Wine Res. 2015, 21, 642-650. [CrossRef]

Publisher's Note: MDPI stays neutral with regard to jurisdictional claims in published maps and institutional affiliations.

(C) 2020 by the authors. Licensee MDPI, Basel, Switzerland. This article is an open access article distributed under the terms and conditions of the Creative Commons Attribution (CC BY) license (http://creativecommons.org/licenses/by/4.0/). 